\section{Alternativas para el aprovechamiento integral de residuos grasos de procesos de fritura}

\section{Alternatives for the integral use of fatty residues from frying processes}

Recibido: 15-feb-17 - Revisado: 30-abr-17

Aceptado: 30-may-17 - Publicado: 30-jul-17

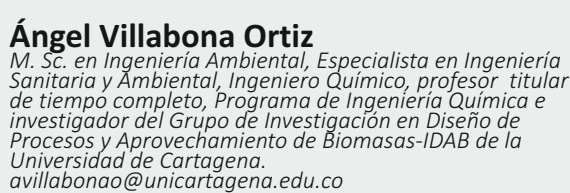

\section{Roy Iriarte Pico}

Especialista en Ingeniería Ambiental de la Universidad de Cartagena, Ingeniero Químico.

\section{Candelaria Tejada Tovar*}

M. Sc. en Ingeniería Ambiental, M.Sc. en Educación especialista en Química Analítica, Ingeniera Química, profesora titular de tiempo completo del Programa de Ingeniería Química, investigadora del Grupo de Investigación en Diseño de Procesos y Aprovechamiento de Biomasa-IDA ctejadat@unicartagena.edu.co

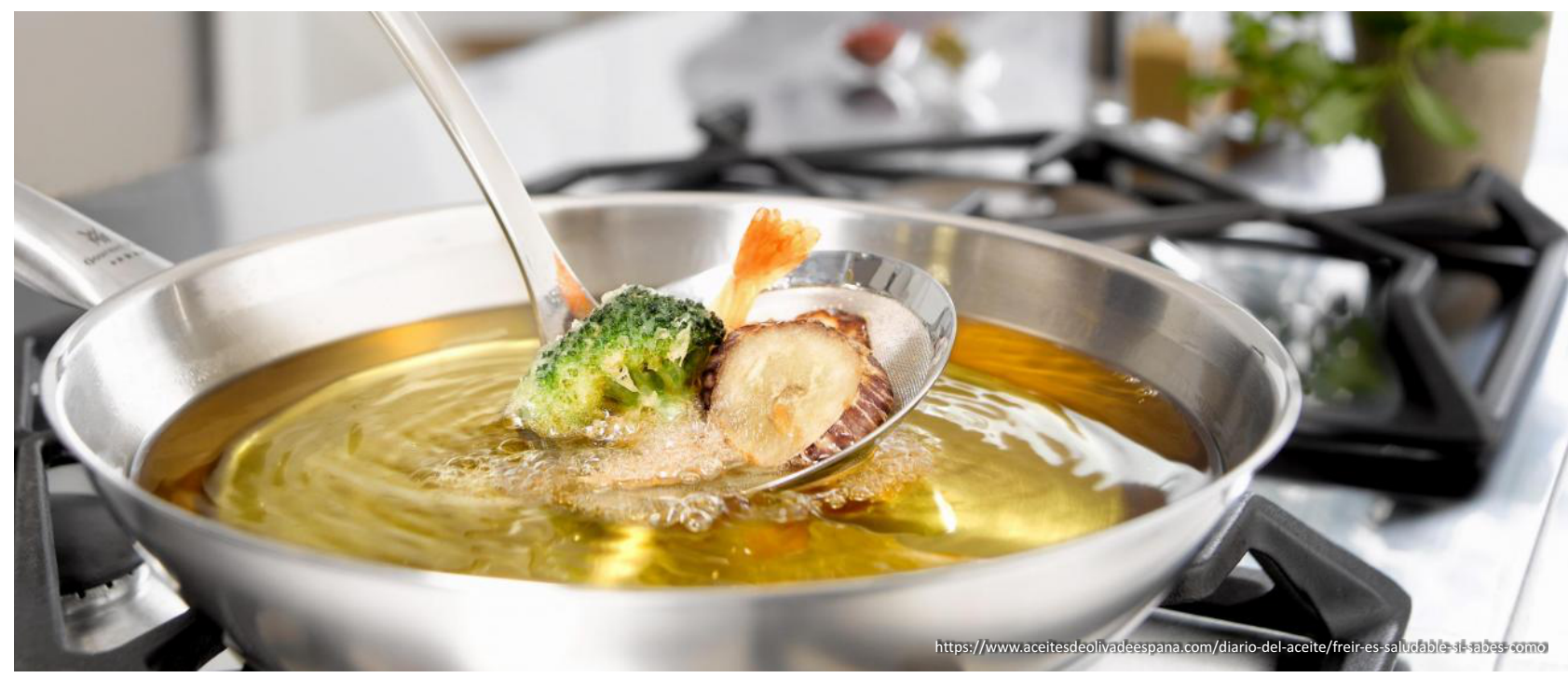

Resumen: El aceite de cocina usado producto de frituras, es un desecho abundante que no cuenta con métodos de disposición apropiados. En este trabajo se realiza una revisión sobre los productos de valor agregado que son factibles de obtener a partir de estos desechos. Se encontró que tienen una alta eficiencia para sintetizar surfactantes, jabón, betún, cera, resinas alquílicas, espuma de poliuretano, entre otros, cumpliendo los criterios de calidad pertinentes con poca inversión. De acuerdo con la bibliografía consultada, el aprovechamiento del aceite de cocina demuestra ser una alternativa rentable y pertinente.

Palabras clave: Aceite de cocina, aprovechamiento integral, biodiésel, biosurfactante.

Abstract: The used cooking oil, product of fritters is an abundant waste which does not have appropriate disposal methods. In this paper is carried out a review about on value-added products that are feasible to obtain from these wastes and found to have high efficiency to synthesize surfactants, soap, shoe polish, wax, alkyd resins, polyurethane foam, between others, fulfilling the criteria relevant quality with little investment. According to the literature, the use of cooking oil proves to be a cost-effective and relevant alternative.

Key words: Cooking oil, comprehensive utilization, biodiesel, biosurfactant. 


\section{INTRODUCCIÓN}

Los aceites vegetales usados son residuos que por su capacidad para formar películas sobre el agua, impiden su oxigenación y dificultan la correcta depuración. En el caso de no haber una depuración total de estos aceites, cuando el agua vuelve al medio ambiente acompañada de residuos del aceite, entonces se produce la contaminación de los ríos, el mar y los acuíferos; así, interfieren en la vida natural y la degradación del entorno, además se proliferan microorganismos perjudiciales para la salud.

Asimismo, la mayoría de los aceites de fritura se vierten en el sistema de alcantarillado de las ciudades. Esta práctica contribuye a la contaminación de ríos, lagos, mares y aguas subterráneas, lo que resulta muy perjudicial para el medio ambiente y la salud humana (Lafont et al., 2011).

Por otro lado, la reutilización excesiva de los aceites de fritura genera elementos cancerígenos como los radicales libres y acrilamidas. Se presume que estos pueden ser reutilizados hasta cinco veces; sin embargo, es mejor no darle ese número de usos, debido a la pérdida de sus propiedades iniciales y sus consecuencias nocivas en la salud (Valdiviezo, 2014).

Según Asociación Colombiana de Aceites y Grasas Comestibles (Asograsas), desde el 2008, se denuncia la existencia de «un cartel pirata del aceite» o mercado negro del aceite. Se trata del sector informal en barrios populares de las ciudades; recogen este desecho generado por algunos restaurantes, hoteles y cafeterías, lo compran para renvalsarlo y luego, lo venden como aceite nuevo, después de someterlo a alteraciones químicas que le devuelven su transparencia. De este modo, ponen en riesgo la salud de las personas, pues al consumirlo, ingieren los radicales libres que se derivan del sometimiento del aceite a diferentes temperaturas.

Esta es la principal razón por la que el Ministerio de ambiente (Minambiente) está evaluando la situación actual del manejo de los aceites comestibles usados; se propone desarrollar políticas que promuevan la creación de operadores especializados en recolección y aprovechamiento de este residuo (Solarte \& Vargas, 2013).

En Colombia, el $35 \%$ de los 162 millones de aceite de cocina vendidos se convierten en residuos desechados a través de sifones y tuberías. Por su parte, el Ministerio de Medio Ambiente y Desarrollo Sostenible afirma que si bien el aceite de cocina usado no se considera residuo peligroso, su mala disposición ocasiona problemas ambientales en redes de alcantarillado, de agua y suelos, debido a la materia orgánica que aporta, que además, tapona las cañerías (Huella Social, 2015).

No obstante, Colombia aún no cuenta con una política de recolección y reciclaje de aceite de cocina, tan solo posee algunas disposiciones constitucionales para grandes consumidores industriales como productores de alimentos. Tener una política sobre esto resulta importante, pues es necesario que el aceite usado de los hogares no termine en el sifón del lavaplatos ni tampoco en manos criminales que le hacen un tratamiento superficial de aclaramiento y lo venden de manera ilegal (Echavarría-Restrepo, 2012).

Por otra parte, las actividades culinarias generan aceites vegetales residuales, los cuales, debido a la falta de normatividad y de conciencia ambiental, son dispuestos de manera directa a las líneas de drenaje municipales o al sistema de recolección de basuras, lo que ocasiona problemas de contaminación (Ibíd., 2015).

La búsqueda de alternativas que contribuyan a disminuir la contaminación por los aceites usados de cocina a través de la utilización comercial resulta de gran importancia. Tales aceites pueden registrarse a una cadena de producción como materia prima para la fabricación de jabón, abono, lubricante, fabricación de 
ceras, pinturas y barniz, o como biocombustible para vehículos. Es indispensable analizar las alternativas para reducir el impacto que causa el vertimiento de aceites al medio ambiente, ya que la mayor parte de la sociedad actual no está dispuesta a disminuir su nivel de consumo (Deng et al., 2010; Liu et al., 2010).

\section{MARCOTEÓRICO}

\subsection{Aceites usados de cocina}

Los aceites vegetales son productos formados por triglicéridos, es decir, ésteres de ácidos grasos y glicerina con pequeñas cantidades de vitaminas, fosfátidos (lecitinas), esteroles, colorantes y agua, principalmente. Los ácidos grasos son sintetizados por plantas y animales y están constituidos por cadenas con un número de carbonos normalmente entre 6 y 18; el ácido oleico, esteárico, palmítico, linoleico son los más comunes (Liu et al., 2010).

Los triglicéridos, componente primordial de los aceites biológicos, son convertidos en ésteres alquílicos por medio de reacciones químicas, con el fin de mejorar sus propiedades de flujo como combustible, en tanto se le retira la glicerina al aceite, factor de aumento de densidad y viscosidad.

Este residuo urbano proviene del uso doméstico en la preparación de alimentos y en lo referente a las orientaciones dadas para los países de la Comunidad Europea, dentro de la normativa actual vigente y según se pone de manifiesto en la Directiva Comunitaria 91/689/CEE, los aceites usados, tanto minerales como vegetales, están considerados como residuos peligrosos, debido a los efectos que pueden tener sobre la salud, como por las consecuencias que pueden afectar el medio ambiente (Solarte \& Vargas, 2013).

\subsubsection{Cambios fisicoquímicos del aceite durante el freído}

Durante el freído, el aceite es sometido a temperaturas entre 160 y $190 \circ \mathrm{C}$. En ocasiones, innecesariamente se eleva por encima del punto de humo en presencia de aire y luz y por tiempos relativamente largos con varias reutilizaciones. Dichas condiciones causan cambios químicos que dependen principalmente de la composición del aceite, ya que al aumentar la temperatura, se aceleran todos los procesos químicos y enzimáticos, por lo que se degradan con rapidez (Cifuentes, 2011).

Durante la utilización de los aceites comestibles, se presentan tres tipos de alteraciones químicas: alteraciones termolíticas, oxidativas e hidrolíticas, causadas por agentes tales como la humedad del equipo de cocción o del material cocinado, el oxígeno del aire y el calor.

Los componentes resultantes de estas alteraciones son: ácidos grasos libres, glicerol, monómeros, dímeros y polímeros oxidados, dímeros no polares, trímeros, epóxidos, componentes volátiles, dímeros y polímeros no oxidados. Además, se presentan cambios en el color, incremento en la viscosidad, en el calor específico, en la tensión superficial y en la tendencia del aceite a formar espuma (Cifuentes, 2011).

\subsection{Impacto ambiental y sanitario de los} aceites usados de cocina

Cuando no se gestionan de la manera adecuada, los aceites usados de cocina traen varias consecuencias en la salud humana y animal, puesto que al exponerlos a altas temperaturas, generan dioxinas, las cuales se consideran agentes cancerígenos (Consejo de Bogotá, 2012). Asimismo, debido a las elevadas temperaturas a las que se someten los aceites para freír alimentos, estos libera elementos cancerígenos como el benzopireno (Valdiviezo, 2014). Por tanto, existe transferencia de dioxinas y ácidos grasos trans al ser humano a través de la carne animal.

Por otro lado, tirar el aceite usado hace que se tapone el drenaje; esto dificulta el paso fluido del agua por las tuberías y por consiguiente, genera trabajo adicional y 
desgaste en las máquinas de filtración y purificación del agua, que luego va a ser distribuida a todos los hogares de la ciudad. Además, el aceite usado crea un ambiente idóneo para la presencia de ratas, cucarachas y bacterias, que llegan a ser un problema para la salud pública.

Si finalmente el aceite usado llega a los ríos y lagos, forma una película que afecta su capacidad de intercambio de oxígeno (Pérez, 2015).

Si el aceite usado de cocina se desecha directamente en los rellenos sanitarios, este contribuye a la generación y permeabilidad de los lixiviados. Aquí se tendría un problema mayor, pues al no controlarse adecuadamente, los lixiviados pueden contaminar los suelos y aguas superficiales y subterráneas, y al ser un proceso contaminante lento, sus efectos no suelen percibirse, sino hasta varios años después (Liu et al., 2010).

El aceite usado de cocina se usa como combustible directo en hornos y calderas, los cuales a altas temperaturas, así como en procesos de incineración incompletos, generan dioxinas, que van directamente a la atmósfera. Entre los posibles efectos de las dioxinas en el aire, podemos mencionar: toxicidad dérmica, inmunotoxicidad, efectos reproductivos negativos y teratgenicidad, así como perturbadores del sistema endocrino.

\section{Alternativas de aprovechamiento de aceites usados de cocina}

Los procedimientos de deposición de aceites usados están generando la degradación del medio ambiente debido a la gran cantidad de contaminantes que producen. Este residuo contamina el agua, ya que cuando es vertido al alcantarillado doméstico o industrial, contamina el recurso hídrico. Además, dicho residuo afecta la salud humana pues después de ser utilizado tres o más veces, se torna potencialmente cancerígeno (Echavarría-Restrepo, 2012; Echavarría, 2012).
Por ello, la reutilización y fabricación de productos con valor agregado a partir de un desecho como el aceite de cocina usado supone una fuente de empleo y una alternativa para la reducción del impacto ambiental y sanitario propiciado por la mala deposición de este residuo. Entre los productos más comunes para tal fin, se encuentran: jabón, betún, biodiésel, velas, cera, surfactantes, tensoactivos, espumas rígidas de poliuretanos, fertilizantes, entre otros.

\subsection{Jabón}

El jabón es un agente limpiador o detergente que se fabrica utilizando grasas animales y/o aceites vegetales, es soluble en agua y por sus propiedades detergentes, se usa comúnmente: en productos destinados a la higiene personal y para lavar determinados objetos o tejidos. Normalmente se presenta en forma de pastilla, en polvo, en crema o en líquido, aunque es sólido en estado natural a temperatura ambiente.

Químicamente, el jabón es la sal sódica o potásica de un ácido graso, obtenido por hidrólisis alcalina de los ésteres contenidos en los materiales grasos. Esta reacción se llama saponificación y es la base de la industria del jabón (Instituto Ecuatoriano de Normalización, INEM, 2012).

En Quito, se produjo jabón a partir de aceites usados de cocina. A través de un diseño experimental factorial 32, se diseñó una planta saponificadora para una producción de 208800 jabones/año bajo las mejores condiciones de reacción; se variaron diferentes temperaturas de reacción y porcentajes de aceite de palma que conforma la mezcla de aceite a saponificar. De esta forma, se encontró que las mejores condiciones de reacción de saponificación de la mezcla aceite vegetal purificado y aceite de palma es de 75 oC y $15 \%$ de aceite de palma, debido a que se obtiene un mayor rendimiento y el producto final cumple con la norma INEN 839 (1981) (Bombón y Albuja, 2014). 
Guerrero, C. (2015), en su proyecto de grado para optar por el título de Ingeniera Química de la Universidad de Almería, España, diseñó una instalación para el tratamiento de aceites vegetales usados procedentes de frituras; también llevó a cabo su posterior conversión en 2276 toneladas de jabón líquido anuales a partir de la reacción de saponificación convencional. Con base en ello y a partir del análisis financiero realizado, se infirió que la producción de jabón es rentable, ya que proporciona utilidades a partir del primer año.

\subsection{Surfactantes}

Los surfactantes o tensoactivos son sustancias anfifílicas, es decir, tienen afinidad polar y apolar en la misma molécula. La parte polar está formada por elementos como O, S, N, P, los cuales aparecen en grupos funcionales como alcohol, éter, éster, ácido, sulfato, sulfonato, fosfato, amina, amida, entre otros. La parte apolar está compuesta en general por hidrocarburos parafínicos o aromáticos; como consecuencia, tienen afinidad por los solventes polares, específicamente el agua, y por los solventes orgánicos, en particular los hidrocarburos, aceites o grasas (Salager \& Fernández, 2014).

A nivel industrial, los surfactantes tienen un gran campo de aplicación como en la producción de detergentes domésticos e industriales, de polímeros, plásticos, pinturas, productos agrícolas, en el sector de alimentos, metalúrgica, farmacéutica, en las industrias petrolera, química y textil, entre otras. En todos los surfactantes, sustancias u objetos delimitados por una superficie son manipulados para obtener una diferencia de tensión superficial que conduzca al desarrollo pertinente de cada proceso (Salager, 1992) (Torres et al., 2011).

En la Universidad Nacional de Colombia, usaron bacilos del género Pseudomonas para metabolizar biosurfactantes. Se evaluaron tres concentraciones iniciales de aceite: $10 \mathrm{~g} / \mathrm{L}, 15 \mathrm{~g} / \mathrm{L}$ y $20 \mathrm{~g} / \mathrm{L}$ con una relación carbono-nitrógeno de 11,6:1. Asimismo, se estableció un método de producción a nivel de laboratorio con erlenmeyer y aireación durante 72 horas a una temperatura de $32 \stackrel{\circ}{\circ}$ y pH 7,00 en medio de cultivo suplementado con $15 \mathrm{~g} / \mathrm{L}$ aceite usado de cocina.

El biosurfactante (producto extracelular) se recuperó mediante centrifugación, acidificación con ácido clorhídrido $(\mathrm{HCl})$ y extracción con cloroformo: metanol (2:1). Para el caso del cloroformo, se usó un rotapavor a 40 ㄷ. Así se obtuvo el biosurfactante puro, el cual se caracterizó en cuanto a tensión superficial y ángulo de contacto.

La tensión superficial mínima alcanzada por el biosurfactante fue $32 \mathrm{mN} / \mathrm{m}$, la concentración micelar crítica (CMC) determinada por conductividad fue de $56,72 \mathrm{mg} / \mathrm{L}$ y el índice de emulsión fue del $52,59 \%$. Por los tanto, se concluyo que tecnológicamente es posible obtener biosurfactantes a partir de aceite de cocina usado (Gómez et al., 2013).

\subsection{Betún y cera para muebles}

La fabricación de betún es un proceso químico que resulta de la interacción de los siguientes productos: ceras, aceites, grasas, pigmentos y disolventes. Estos productos se calientan y se mezclan para obtener la proporción adecuada. Luego de la mezcla, se vierte dentro de recipientes, para así, lograr un cambio en su estado: pasa de líquido a sólido. Posterior a ello, el betún se encuentra listo para su comercialización.

Por otro lado, la elaboración de cera para muebles se lleva a cabo con la mezcla de grasas y aceites usados, cera de abejas, trementina y aceite mineral; disueltos los productos, deben ser calentados y luego expuestos a temperatura ambiente para que se solidifique el producto final. De este modo, se consigue que la cera tenga una buena consistencia y que pueda, a su vez, proporcionar un buen brillo (Rodríguez y Erazo, 2011). 


\subsection{Fertilizantes}

Un proyecto de investigación del Centro de Edafología y Biología Aplicada del Segura (CEBAS) titulado «Demostración del ciclo de residuos cero mediante la completa valorización de los residuos del refinado de aceites vegetales usados» y finalizado en febrero de 2013, surgió para dar solución a los residuos generados en el proceso de conversión de los aceites vegetales usados (AVU) de uso doméstico en biodiésel. Se ha logrado obtener energía y fertilizantes utilizando estos $A V U$, con el avance añadido de eliminar en el proceso todos los residuos (Unión Europea, 2013).

\subsection{Biodiésel}

La elaboración de biodiésel a partir de aceites vegetales usados es una aplicación emergente que se ha desarrollado rápidamente y con la que se están realizando diversas pruebas piloto en varios países. El proceso de tratamiento de los AVU para la aplicación como biodiésel se basa en un conjunto de reacciones químicas, un proceso de transesterificación, donde actúan: aceite vegetal o grasa, un alcohol (metanol) y un catalizador alcalino. Es este proceso, el $85 \%$ se convierte en biodiésel y el $15 \%$, en glicerol (Uzun et al., 2012).

En el proceso de transesterificación, se remueve la glicerina de los triglicéridos y se sustituye por el alcohol usado para el proceso de conversión, el cual disminuye la viscosidad, pero mantiene el número de cetano y el poder calorífico (Navas et al., 2015).

Diferentes autores han estudiado el uso de aceites residuales de cocina para la elaboración de biodiésel. López et al., (2015) evaluaron la obtención de este tipo de combustible a partir de la catálisis con hidróxido de sodio y potasio, con tres relaciones molares de metanol-aceite $(6: 1,9: 1$ y $12: 1)$, dos catalizadores $\mathrm{KOH}$ y $\mathrm{NaOH}$ y dos porcentajes de catalizador, $1 \%$ y $2 \%$. Así, se realizó un diseño factorial por duplicado a temperatura constante de $60^{\circ} \mathrm{C}$ y con un tiempo de reacción de dos horas.
Se determinó el contenido de metilésteres y de mono, di- y triglicéridos mediante cromatografía de gases. Las mejores condiciones para $\mathrm{NaOH}$ y $\mathrm{KOH}$ como catalizadores se obtuvieron cuando el porcentaje de catalizador fue 1 \% y la relación molar alcohol-aceite fue 12:1. En estas condiciones, se obtuvo una conversión de $64,1 \%$, un rendimiento de $98 \%$ y un porcentaje de metilésteres de 99,1 \% cuando el catalizador fue $\mathrm{NaOH}$. Con $\mathrm{KOH}$ la conversión fue de $63,2 \%$; el rendimiento, $88 \%$ y el porcentaje de metilésteres, 98,4\%. Lo anterior muestra un alto rendimiento de la reacción al emplear aceite usado, por lo tanto, su implementación industrial dependerá de la evaluación económica.

Sirajunnisa y Surendhiran (2015) estudiaron la metodología de respuesta asistida de la producción de biodiésel a partir de aceite de cocina usado utilizando la enzima encapsulada mixta. Las condiciones óptimas requeridas para generar biodiésel fueron: $2 \mathrm{~g}$ de carga enzima, relación de 1:12 entre aceite y acetato de metilo, 60 horas de tiempo de reacción y temperatura de $35^{\circ} \mathrm{C}$, con un máximo de $93,61 \%$ de biodiésel.

Las perlas de lipasa inmovilizada se mantienen estables sin ningún cambio en su función y estructura, incluso después de 20 ciclos. Lugo et. al (2014) usaron $\mathrm{KOH}$ como catalizador durante dos horas, a través de un reflujo con metanol; variaron la temperatura de reacción, la relación molar aceite-metanol y la concentración de catalizador, con el fin de establecer condiciones óptimas de reacción. La mayor conversión en biodiésel, $93,7 \%$, se obtuvo a temperatura de $65^{\circ} \mathrm{C}$, relación molar de 1:6 (Aceite:Metanol) y una concentración entre $0,5-2 \%$ de $\mathrm{KOH}$. El producto obtenido fue caracterizado mediante la determinación de densidad, viscosidad cinemática, viscosidad absoluta, API, índice de acidez, punto de inflamación, contenido de agua y sedimentos y espectroscopia IR, de tal manera que se cumpliera con las especificaciones de la Norma A.S.T.M D 6751 para biodiésel y comparables a las obtenidas para un diesel comercial. 


\subsection{Espuma rígida de poliuretano}

También, autores como Tahir y colaboradores en 2016 investigaron la síntesis de una espuma rígida de Poliuretano (PU) a partir de aceites usados de cocina. Para ello, se filtró el aceite y se adsorbió mediante el uso de bagazo de caña de carbón activado con el fin de purificar el aceite. Luego de éste paso, se usó el aceite filtrado para sintetizar poliol a través de una reacción de transesterificación. Posteriormente, el poliol se combinó con otros productos químicos en diversas proporciones para formar espuma de poliuretano rígida.

El espectro FTIR de poliol mostró la formación de pico de absorción $\mathrm{OH}$ y fue apoyada por el aumento en el índice de hidroxilo desde 0 hasta 148,79 mg de $\mathrm{KOH} / \mathrm{g}$ después de la reacción. La formación de la columna vertebral de uretano vínculos en espuma de poliuretano se confirmó usando FTIR.

Las propiedades de la espuma de PU dependen altamente de la composición química. La densidad y la resistencia a la compresión de 60: 54: 90: 40 de glicerol: agua: poliol: espuma de poliuretano de amina son $277,7 \mathrm{~kg} / \mathrm{m} 3$ y 0,10 MPa, respectivamente; esto muestra el potencial del aceite de cocina usado para sintetizar PU.

\section{CONCLUSIONES}

La revisión realizada reveló que el aceite de cocina, considerado un residuo clasificado como producto de desecho, puede ser utilizado como una materia prima para la producción de diversos productos con potencial de industrialización, tales como: biocombustible (biodiésel), betún, surfactantes, jabón, espuma de poliuretano y fertilizantes. De esta forma, se disminuye el impacto ambiental y se aprovecha el valor agregado de un residuo considerado actualmente como desperdicio.

Otro aporte del presente trabajo consiste en mostrar el gran potencial de valorización de este tipo de residuos para la generación de empresas. Además, se evidenció la importancia, desde el punto de vista ambiental, que representaría el aprovechamiento de aceites de cocina usados, los cuales en su gran mayoría, son arrojados sin tratamiento alguno a los sistemas de drenaje municipal o en el peor de los casos, son tratados mínimamente para su reúso y comercialización ilegal en diversas actividades. 


\section{REFERENCIAS}

Bombón N. \& Albuja, M. (2014). Diseño de una planta de saponificación para el aprovechamiento del aceite vegetal de desecho. Revista Politécnica, 34(1), 1-10

Cifuentes, G. M. (2011). Obtención de biodiésel a partir de aceite usado de cocina por transesterificación en dos etapas, con dos tipos de alcoholes (Tesis de Maestría). Universidad Libre, Bogotá, Colombia.

Concejo de Bogotá (2012). Proyecto de Acuerdo 292 de 2012, por el cual se establecen mecanismos para la recolección de aceites vegetales usados para prevenir la contaminación ambiental e hídrica en Bogotá D.C.

Deng, S., Lammers, P., Patil, P. y Rhodes, J. (2010). Conversion of waste cooking oil to biodiesel using ferric sulfate and supercritical methanol processes. Fuel, 89(2), 360-364.

Echavarría, J.I. (2012) Aceites vegetales usados y principios del derecho ambiental. Ambiente Jurídico, 14, 66-84.

Echavarría-Restrepo, J. (2012). El desarrollo sostenible y el reciclaje del aceite usado de cocina a la luz de la jurisprudencia y el ordenamiento jurídico colombiano. Producción + Limpia, 7(1), 109-112.

Gómez, M., Velázquez, L., Barragán, R., MalagónRomero, D. (2013). Obtención y caracterización de un biosurfactante sintetizado por Pseudomonas sp. usando aceite de cocina como sustrato. Intekhnia, 7(2), 133-141.

Guerra, J. M. Proyecto de Ley No. 061 de 2014 Senado, Bogotá, Colombia, 12 de Agosto de 2014.

Guerrero, C. (2015). Diseño de una planta de fabricación de jabón a partir de aceites vegetales usados (Tesis de pregrado). Universidad de Almería, La Cañada, España.

Huella Social (2015, Mayo, 4). Recicle el aceite usado de cocina y evite riesgos para su mesa. El Tiempo. Recuperado de:

http://www.eltiempo.com/archivo/documento/CMS15683577.

Instituto Ecuatoriano de Normalización (INEN). NTE INEN 0846: (Spanish): Agentes tensoactivos. Jabón abrasivo en barra. (2012). Requisitos. Consultado: 06 de Octubre de 2017. Disponible en: https://ia801901.us.archive.org/3/items/ec.nte.0846 .1982/ec.nte.0846.1982.pdf.

Lafont, J.J., Páez, M., \&Torres, Y.C. (2011). Análisis químico de mezclas biodiésel de aceite de cocina usado y diésel por Espectroscopía Infrarroja. Información Tecnológica, 22(4), 35-42.

Liu, S., McDonald, T. \& Wang, Y. (2010). Producing biodiesel from high free fatty acids waste cooking oil assisted by radio frequency heating. Fuel, 89(10), 2735-2740.

López, L., Bocanegra, J., y Malagón-Romero, D. (2015). Obtención de biodiesel por transesterificación de aceite de cocina usado. Ing. Univ., 19(1), 155-172.

Lugo, M., Bergolla, D. \& Rodríguez, K. (2014). Producción de biodiésel a partir de la transesterificación de aceites vegetales residuales con KOH como catalizador. Ciencia, 22(2), 121-128.

Navas, M., Ruggera, J., Casella, M. (2015). Transtereficación de aceites vegetales con metanol mediante catálisis heterogénea. Investigación Joven Revista Electrónica, 2(2), 53-54.

Perez, M.A. (2015, Abril, 1). Primer vuelo de un avión que utiliza aceite de cocina reciclado como 
combustible. Blogthinkbig.com. Recuperado de: http://blogthinkbig.com/aceite-de-cocina-comocombustible-en-aviones/

Rodríguez, C.A.P., \& Erazo, J.G. (2011). Aprovechamiento de los residuos grasos generados en los restaurantes y comidas rápidas de Pereira. Scientia et Technica, 1(47), 264-269.

Salager, J. (1992). El mundo de los surfactantes. Mérida, Venezuela: Universidad de los Andes. Recuperado de: http://www.firp.ula.ve/ archivos/cuadernos/S311A.pdf.

Salager, J. y Fernández, A. (2014). Módulo de enseñanza en fenómenos interfaciales. Venezuela: Universidad de los Andes y Ministerio de Ciencia y Tecnología. Re c u p e r a do d e : http://www.firp.ula.ve/site/es/cuadernos-firpgratuitos

Tahir, S.M., Salleh, W.N.W., Hadid, N.S.N., Enderus, N.F. \& Ismail, N.A. (2016). Synthesis of Waste Cooking OilBased Polyol Using Sugarcane Bagasse Activated Carbon and Transesterification Reaction for Rigid Polyurethane Foam. Materials Science Forum, 846, 690-696.

Torres, L., Moctezuma, Avendaño, JR, Muñoz, A., Gracida, J. (2011). Comparison of bio- and synthetic surfactants for EOR. Journal of Petroleum Science and Engineering, 76(1-2), 6-11.

Sirajunnisa, A. \& Surendhiran, D. (2016). Response surface methodology assisted biodiésel production from waste cooking oil using encapsulated mixed enzyme. Waste Manag., 47, Part A., 98-104.

Solarte, N., \& Vargas, M. C. (2013). Diseño de las estrategias de recolección del aceite de cocina usado para su reutilización en la producción de biodiésel en 4 barrios de la ciudad de Cali (Tesis de pregrado).
Universidad Autónoma de Occidente, Santiago de Cali, Colombia.

Unión Europea (2013). VALUVOIL- Demonstration of zero waste cycle by the complete valorization of residues from refining of used vegetable oils. Recuperado de:

http://ec.europa.eu/environment/life/project/Projec ts/index.cfm?fuseaction=search.dspPage\&n_proj_id $=3652$

Uzun, B., Kilic, M., Ozbay, N., Putun, A., \& Putu, E. (2012). Biodiésel production from waste frying oils: Optimization of reaction parameters and determination of fuel properties. Energy, 44, 347-351.

Valdiviezo, N. (2014). Análisis del tipo de aceite y tiempo de fritura en la vida útil del Snack de Malanga (Xanthosoma sagittifolium) procedente del Tena (Tesis de pregrado). Universidad técnica de Ambato, Ámbato, Ecuador. 